\title{
Most of the Abundant Protein Fractions of Embryonic Cerebrospinal Fluid are Produced Out of the Brain Anlagen
}

\author{
M. Parvas, M. Rius and D. Bueno*
}

Departament de Genètica, Facultat de Biologia, Universitat de Barcelona, Av. Diagonal 645, 08028 Barcelona, Catalonia, Spain

\begin{abstract}
The microenvironment of the central nervous system is important for neuronal function and development. During the early stages of embryo development the cephalic vesicles are filled by embryonic cerebrospinal fluid, a complex fluid containing different protein fractions, which contributes to the regulation of the survival, proliferation and neurogenesis of neuroectodermal stem cells. The protein content of embryonic cerebrospinal fluid from chick and rat embryos at the start of neurogenesis has already been determined. Most of the identified gene products are thought to be involved in the regulation of developmental processes during embryogenesis. However, due to the crucial roles played by embryonic cerebrospinal fluid during brain development, the embryological origin of the gene products it contains remains an intriguing question. According to the literature most of these products are synthesised in embryonic tissues other than the neuroepithelium. In this study we examined the embryological origin of the most abundant embryonic cerebrospinal fluid protein fractions by means of slot-blot analysis and by using several different embryonic and extraembryonic protein extracts, immunodetected with polyclonal antibodies. This first attempt to elucidate their origin is not based on the proteins identified by proteomic methods, but rather on crude protein fractions detected by SDS-PAGE analysis and to which polyclonal antibodies were specifically generated. Despite some of the limitations of this study, i.e. that one protein fraction may contain more than one gene product, and that a specific gene product may be contained in different protein fractions depending on post-translational modifications, our results show that most of the analysed protein fractions are not produced by the cephalic neuroectoderm but are rather stored in the egg reservoir; furthermore, few are produced by embryo tissues, thus indicating that they must be transported from their production or storage sites to the cephalic cavities, most probably via embryonic serum. These results raise the question as to whether the transfer of proteins from these two embryo compartments is regulated at this early developmental stage.
\end{abstract}

\section{MAIN TEXT}

The development of the CNS from relatively simple anlagen involves the simultaneous and interdependent action of various developmental mechanisms, including the establishment of positional identities, morphogenesis and histogenesis. It has been demonstrated that diffusible molecules, such as growth factors and morphogens secreted locally by organising centres, regulate these processes by controlling neighbouring cells in an autocrine/paracrine manner [1], and that they regulate the expression of a number of transcription factors through which complex molecular and genetic networks are established.

The architecture of the brain primordium reveals the existence of an internal cavity, the brain cavities, which in foetuses and adults become the ventricular system of the brain, containing cerebrospinal fluid (CSF). It has been reported that embryonic cerebrospinal fluid (E-CSF), which contacts the apical surface of all the neuroepithelial cells of the cephalic vesicles, with which it establishes a physiologically sealed system, plays important roles in brain development: (1) it exerts positive pressure against the neuroepithelial walls to generate expansive force [2-5]; (2) it contributes to the regulation of the survival, proliferation and neural differentiation of neuroepithelial progenitor cells $[4,6,7]$; (3) it

\footnotetext{
*Address correspondence to this author at the Departament de Genètica, Facultat de Biologia, Universitat de Barcelona, Av. Diagonal 645, 08028 Barcelona, Catalonia, Spain; E-mal: dbueno@ub.edu
}

collaborates with the isthmic organiser in regulating mesencephalic gene expression during the establishment of the anterior CNS pattern [8]; and (4) it controls the formation of layers of neurones in the cerebral cortex, as well as neuronal proliferation at foetal stages $[5,7,9]$.

It has recently been shown that at the moment of maximum proliferation and differentiation of neuroepithelial progenitor cells, the E-CSF proteomes of both avians (chick, at developmental stage HH24 according to Hamburger and Hamilton [10], as depicted in Bellairs and Osmond [11] and mammals (rodents, at 12.5-12.7 days post coitum; and humans, at CS 19-20) include molecules whose roles during development in systems other than E-CSF are related to the functions reported for this fluid [12-14]. In chick embryos [13], these molecules include gene products related to the extra-cellular matrix and the regulation of osmotic pressure, regulators of lipid metabolism, i.e. apolipoprotein AI and AIV, molecules involved in vitamin transport (e.g. retinolbinding protein and transthyretin), and proteins related to cell quiescence and death, among others. Moreover, we have also recently reported the presence of FGF2 within the ECSF and its role in neural differentiation [15].

Due to the crucial roles played by E-CSF during brain development, one intriguing question is the embryological origin of the gene products it contains. According to the literature, most of the molecules identified in chick E-CSF are not produced by the neuroectoderm itself but rather by other 
embryonic structures; alternatively, they are stored in the yolk or white of the egg and are consequently uptaken by the chorioallantois membrane [13]. Interestingly, a significant number of proteins identified within E-CSF are also present in blood plasma, although there are important quantitative and qualitative differences between these two fluids [16]. In this study, we examined the embryological origin of the most abundant E-CSF protein fractions at $\mathrm{HH} 24$ by means of slotblot analysis and by using several different embryonic and extraembryonic protein extracts, this being achieved through the generation of polyclonal antibodies to the most abundant protein fractions. This study is not based on the proteins identified by proteomic methods but rather on crude protein fractions as detected by SDS-PAGE analysis [16].

The developmental stage from which we obtained E-CSF was chosen for two main reasons. First and most important, this stage falls within the period at which the embryos show the highest rate of neural stem cell proliferation, and at which the process of neurogenesis starts. This is also the reason by which all currently available data on the role of chick E-CSF on early CNS formation have been focused at HH24 (E4). Second, at stages earlier than HH23 the cephalic cavities are too small to obtain enough E-CSF to perform the analysis. In this way, this work contributes some new data on the origin of the E-CSF protein content at the developmental stage showing the highest rate of neural stem cell proliferation and the starting of neurogenesis.

Fertile chicken eggs were incubated at $38^{\circ} \mathrm{C}$ in a humidified atmosphere to obtain chick embryos at the desired developmental stage, i.e. at E4 (HH24) (E is for embryonic day according to the beginning of incubation). To generate polyclonal antibodies, $1 \mathrm{ml}$ of E-CSF obtained from 200 embryos at $\mathrm{HH} 24$ was in SDS-polyacrylamide gel electrophoreses (three gels at $7.5 \%$ and three gels at $12 \%$ ) and subsequently stained with Silver Stain Kit (Bio-Rad). After identifying the 21 most abundant E-CSF protein fractions previously described [16], they were cut with a scalpel and homogenised in PBS by sonication. For mice immunisation, the protocol described by Harlow and Lane [17] was followed. Briefly, $300 \mu \mathrm{l}$ of each preparation was injected intraperitoneally into eight-week old BALB/C female mice (two mice for each protein fraction). The injection was repeated twice, at a twoweek interval. Finally, mice were injected daily for three days, blood was removed by post-mortem intracardiac injection, and serum recovered after centrifugation. Polyclonal antibodies were purified by protein-A columns (Sigma, 82487). All animal experimentation was performed according to local animal welfare regulations. The specificity of the obtained antibodies was checked by western-blot analysis to the protein fractions used to generate them using standard protocols (not shown).

For slot-blot analysis, embryonic samples were applied to nitrocellulose membrane (Hybond-N) using a Micro-sample Filtration Manifold (Schleicher \& Schuell, SRC 072/0) connected to a vacuum pump. Samples were as follows: E-CSF, embryonic serum, and protein extracts from chorioallantois membrane, total embryo, dorsal cephalic neuroectoderm from which most of the subjacent mesenchyma had been dissected off, yolk and white of the egg. All samples were taken from fertile eggs after four days of incubation. After extensive washes in PBS, tissue samples were homogenised in PBS. Yolk and white of the egg were diluted 1/10 in PBS. After sample application, membranes were dried to fix the proteins and the presence of molecules was detected with the raised polyclonal antibodies diluted at 1/5,000 in PBS. As secondary antibody a goat anti-mouse conjugated to HRP (at 1/2,000; Sigma, A0168) was used.

It is important to note that total embryonic tissues contained some remaining E-CSF and embryonic serum within the neural tube and blood vessels, respectively, and that chorioallantois membrane also contained some remaining embryonic serum within the blood vessels. Ventral telencephalic and mesencephalic tissues were not used due to the difficulty of dissecting them from other embryonic tissues, for example, from the underlying mesenchyma.

The logic behind this experiment is as follows: (1) protein fractions detected either in the yolk or white of the egg as well as in E-serum are most probably taken from the egg reservoir by the chorioallantois membrane and transported to the E-CSF by means of E-serum; (2) protein fractions detected in the chorioallantois membrane and E-serum but in neither the yolk nor white of the egg are those eligible to be produced by the chorioallantois membrane and transported to the E-CSF by means of E-serum; (3) protein fractions detected in the total embryo tissues but in neither the egg reservoir nor the neuroectoderm are those eligible to be produced by embryonic tissues or organs other than the dorsal neuroectoderm; and (4) protein fractions detected mostly in the neuroectoderm are those eligible to be produced by this tissue. In all cases, proteins must be detected within the ECSF as this is the source of the antigens used for immunisation. It is important to note some of the limitations of this study, i.e. that one protein fraction may contain more than one gene product, and that a specific gene product may be contained in different protein fractions depending on posttranslational modifications. Similarly, a specific polyclonal antibody may recognise epitopes present in different gene products.

Protein fractions obtained by SDS-PAGE were named A to $\mathrm{U}$ according to Gato et al. [16] (Fig. 1A). The mass spectrometry analysis of these bands was not conclusive most probably due to the presence of some underrepresented gene products in the protein fractions, which yield some peptides that masked the identification of the tryptic digests. Chick ECSF proteins identified by mass spectrum analysis from a 2 DE have been described in Parada et al. [13]. For the assigned nature of $A$ to $U$ protein fractions on the basis of their SDS-PAGE molecular mass, see Gato et al. [16].

This experiment showed that most of the analysed protein fractions $(76.19 \%)$ were present either in the yolk or white of the egg, and only $23.81 \%$ were restricted to embryo tissues (Fig. 1B). All protein fractions that were not present in either the yolk or white of the egg (i.e. fractions E, I, N, O and $\mathrm{U}$ ) were also absent in chorioallantois extracts, further indicating that they were produced by embryo tissues. Similarly, all protein fractions that were present in the yolk or white of the egg were also present in the chorioallantois extracts and in E-serum, suggesting that this is the route they follow to reach E-CSF. As expected, all protein fractions including those coming from the egg reservoir and those produced by the embryo tissues were detected in total embryo protein extract. No proteins produced exclusively by 
A

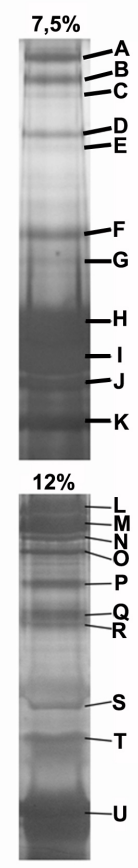

B
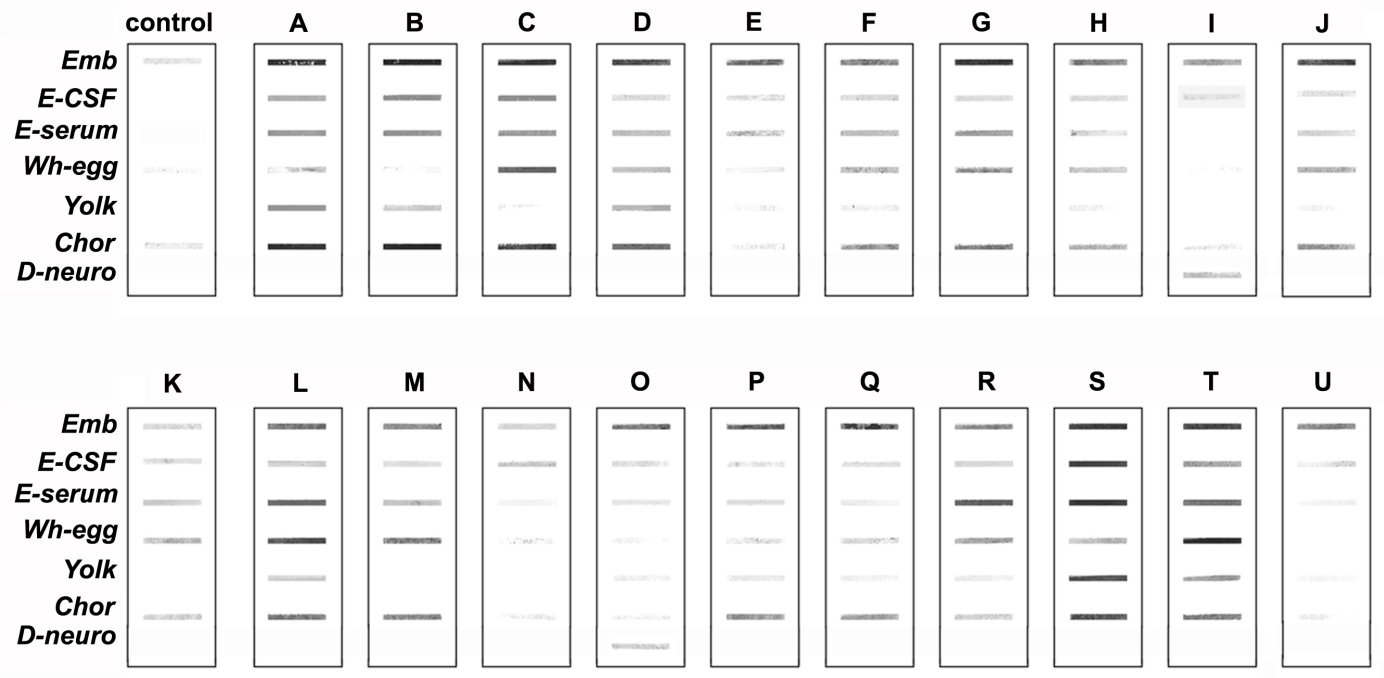

Fig. (1). Embryological origin of the most abundant E-CSF protein fractions at the beginning of primary neurogenesis. A) Silver staining of SDS-PAGE of E-CSF at E4, showing the 21 most abundant protein fractions (named A to U according to Gato et al., 2004). B) Slot-blot analysis of several different embryonic and extraembryonic protein extracts with the 21 polyclonal antibodies to the corresponding 21 most abundant protein fractions. Control was made with mouse antiserum obtained before immunisation. The intensity of the negative control was considered as normalised background. Abbreviations: Emb, total embryo extract (which includes some remaining E-CSF from the neural tube and some remaining E-serum from the blood vessels); E-CSF, embryonic cerebrospinal fluid; E-serum, embryonic serum; Wh-egg, white of the egg; Yolk, yolk of the egg; Chor, chorioallantois membrane; D-neuro, dorsal neuroectoderm.

the chorioallantois membrane were detected, as the polyclonal antibodies which immunoreacted with chorioallantois membrane extract also immunoreacted with either the yolk or the white or the egg. With respect to proteins produced by embryo tissues, fractions I and $\mathrm{O}$ were detected in neuroectodermal extracts but fractions $\mathrm{N}$ and $\mathrm{U}$ were not, thus suggesting that the former were produced by the neuroectoderm and the latter by other embryonic tissues, which could include ventral neuroectoderm as this was not included in the analysis.

Despite the abovementioned limitations of this study, which do not allow assigning the precise embryological origin to particular known proteins, the overall results indicate that most of the analysed protein fractions are not produced by the neuroectoderm, and thus they may be transported from their production or storage sites to the cephalic cavities, most probably via E-serum. The extraneural origin of most of these protein fractions, together with the crucial roles exerted by the analysed gene products of this fluid and the differences reported for E-CSF and embryonic serum composition $[16,18]$, raise the question as to whether the transfer of these proteins from these two embryo compartments is regulated, thus suggesting the existence of some kind of bloodCSF interface for protein transfer. In this regard, it is known that choroids plexuses, which fulfil this function in foetuses and adults, develop later in development, i.e. according to Bellairs and Osmond [11] they are first detected at the morphological level at E7 in chick embryos. The particular analysis of these issues merits further experimentation.

\section{COMPETING INTERESTS}

The authors declare that they have no competing interests.

\section{AUTHOR CONTRIBUTIONS}

MP contributed to slot-blot analysis, obtaining and purifying the antibodies and drafted the manuscript. MR contributed to slot-blot analysis. DB was responsible for conceiving, designing and coordinating the study; he also produced the final draft of the manuscript and obtained and purified the polyclonal antibodies. All authors have read and approved the final version of the manuscript.

\section{ACKNOWLEDGEMENTS}

The authors thank Victor Hernández for help in immunizing mice; and Angel Gato and Pedro Martín for SDSPAGE protein obtaining and Fig. (1A). This work was supported by the Ministerio de Sanidad y Consumo, Instituto de Salud Carlos III (Grant 0915), co-funded by the European Community (FEDER), and through Accions Especials 2006 \& 2007 from the University of Barcelona (to DB).

\section{REFERENCES}

[1] Bally-Cuif, L.; Boncinelli, E. BioEssays, 1997, 19, 27.

[2] Alonso, M.I.; Gato, A.; Moro, J.A.; Martin, P.; Barbosa, E. Cells Tissues Organs, 1999, 165, 1.

[3] Desmond, M.E.; Jacobson, A.G. Dev. Biol., 1977, 57, 188. 
[4] Gato, A.; Moro, J.A.; Alonso, M.I.; Bueno, D.; De La Mano, A.; Martín, C. Anat. Rec. Part A, 2005, 284a 475.

[5] Miyan, J.A.; Nabiyouni, M.; Zendah, M. Can. J. Physiol. Pharmacol., 2003, 81, 317.

[6] Mashayekhi, F.; Draper, C.E.; Bannister, C.M.; Pourghasem, M.; Owen-Lynch, P.J.; Miyan, J.A. Brain, 2002, 125, 1859.

[7] Owen-Lynch, P.J.; Draper, C.E.; Mashayekhi, F.; Bannister, C.M.; Miyan, J.A. Brain, 2003, 126, 623.

[8] Parada, C.; Martín, C.; Alonso, M.I.; Moro, J.A.; Bueno, D.; Gato, A. J. Neurosci. Res., 2005, 82, 333.

[9] Miyan, J.; Zendah, M.; Mashayekhi, F.; Owen-Lynch, P.J. Cereb. Fluid Res., 2006, 3, 2.

[10] Hamburger, V.; Hamilton, H.L. J. Morphol., 1951, 88, 49.

[11] Bellairs, R.; Osmond, M. Atlas of Chick Development. Elsevier Academy Press, London. 2005.
[12] Parada, C.; Gato, A.; Bueno, D. J. Proteome Res., 2005, 4, 2420.

[13] Parada, C.; Gato, A.; Aparicio, M.; Bueno, D. Proteomics, 2006, 6, 312.

[14] Zappaterra, M.D.; Lisgo, S.N.; Lindsay, S.; Gygi, S.P.; Walsh, C.A.; Ballif, B.A. J. Proteome Res., 2007, 6, 3537.

[15] Martin, C.; Bueno, D.; Alonso, M.I.; Moro, J.A.; Callejo, S.; Parada, C.; Martín, P.; Carnicero, E.; Gato, A. Dev. Biol., 2006, 297,402

[16] Gato, A.; Martin, P.; Alonso, M.I.; Martin, C.; Pulgar, M.A.; Moro, J.A. J. Exp. Zool. Part B, 2004, 301, 280.

[17] Harlow, E.; Lane, D. Antibodies. A laboratory manual, Cold Spring Harbour Laboratory. Cold Spring Harbour, New York. 1988.

[18] Parada, C.; Parvas, M.; Bueno, D. Curr. Proteomics, 2007, 4, 89.

Received: November 30, 2007

(c) Parvas et al.; Licensee Bentham Open.

This is an open access article distributed under the terms of the Creative Commons Attribution License (http://creativecommons.org/licenses/by/2.5/), which permits unrestrictive use, distribution, and reproduction in any medium, provided the original work is properly cited. 